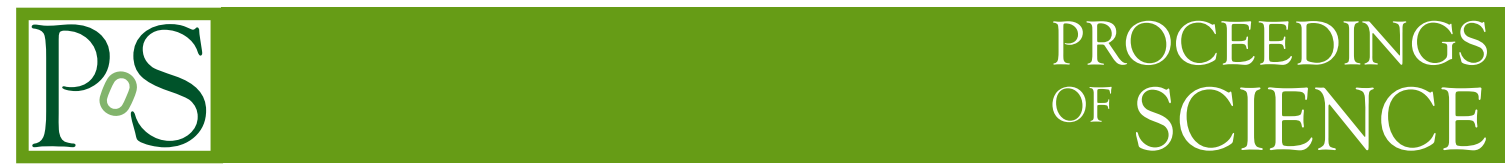

\title{
Going Deeper, Wider and Wideband
}

\author{
Emil Lenc* \\ CSIRO Astronomy and Space Science \\ E-mail: Emil.Lencecsiro.au
}

To better understand the early Universe, astronomers frequently push the limits of available instrumentation and technology to go both deeper and wider. While the rewards of taking such a path can be tremendous, the journey is often difficult and fraught with obstacles and traps. With the Compact Array Broadband Backend (CABB) online now for well over a year I will discuss some of the "adventures" that have been had in dealing with and reducing CABB data for deep and wide imaging.

ISKAF2010 Science Meeting - ISKAF2010

June 10-14, 2010

Assen, the Netherlands

${ }^{*}$ Speaker. 


\section{Introduction}

The Compact Array Broadband Backend upgrade (CABB, Wilson et al. 2010, in prep.) provides a new back end for the Australia Telescope Compact Array (ATCA). The upgrade results in a 16-fold increase in bandwidth at $6 \mathrm{~cm}, 3 \mathrm{~cm}$ and at millimetre wavelengths, while also providing $600 \mathrm{MHz}$ at $20 \mathrm{~cm}$ and $500 \mathrm{MHz}$ at $13 \mathrm{~cm}$ (simultaneous $20 \mathrm{~cm}$ and $13 \mathrm{~cm}$ observations are currently not available), see Figure 1 . At all bands, full stokes and $1 \mathrm{MHz}$ channels are available as standard, so in effect CABB has been delivering $8 \mathrm{GHz}$ total bandwidth since normal operation began in April 2009 and thus has provided valuable experience for upcoming instruments such as the eVLA and eMERLIN.
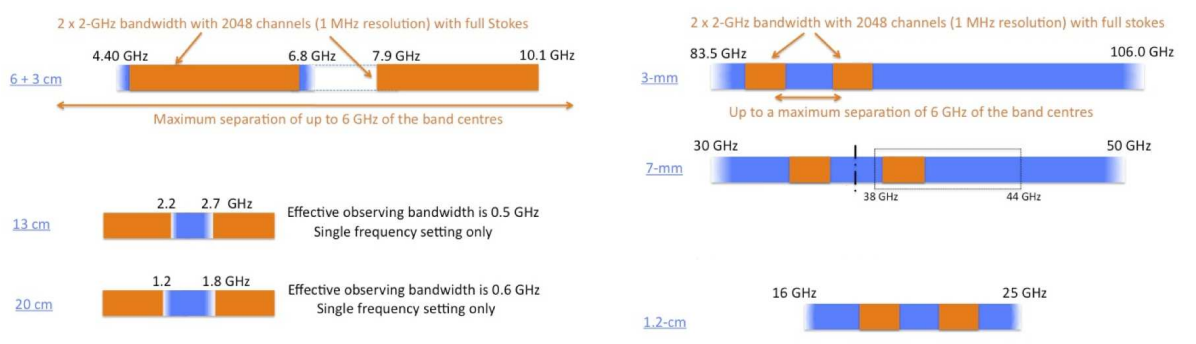

Figure 1: ATCA observing bands at centimetre (left) and millimetre (right) wavelengths.

\section{CABB}

\subsection{Improved $u-v$ coverage}

The wide bandwidths available with $\mathrm{CABB}$ greatly improve $u-v$ coverage at centimetre wavelengths when using multi-frequency synthesis. This is particularly so at $3 \mathrm{~cm}$ and $6 \mathrm{~cm}$ where the two CABB bands overlap in $u-v$ space to provide near-complete coverage, see Figure 2.
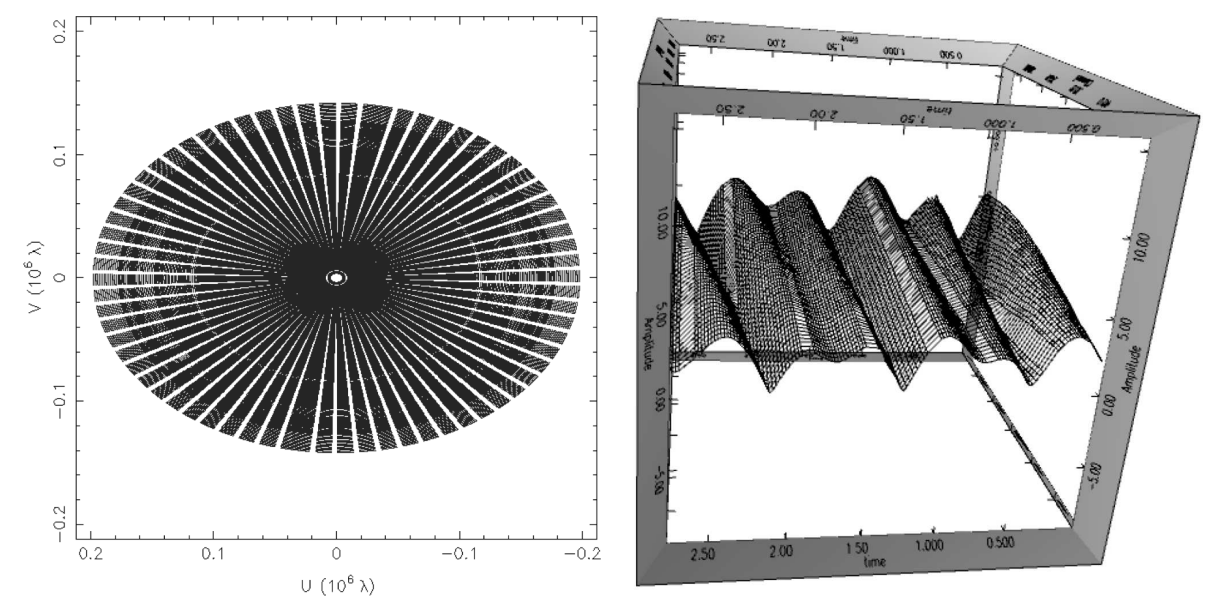

Figure 2: (Left) Multi-frequency synthesis $u-v$ coverage from a single epoch observation at $3 \mathrm{~cm}$ and $6 \mathrm{~cm}$. (Right) Structure seen in visibilities both temporally and spectrally, note that the frequency axis is shown going into the page. 
The improved $u-v$ coverage and wider band results in increased dynamic range and fidelity. An example of this can be seen with early observations of Circinus made during the scientific commissioning of $\mathrm{CABB}$. A single $6 \mathrm{~cm}$ CABB observation resulted in a three-fold improvement in sensitivity compared to an earlier five-epoch/five-configuration pre-CABB observation of the same source (Figure 3).
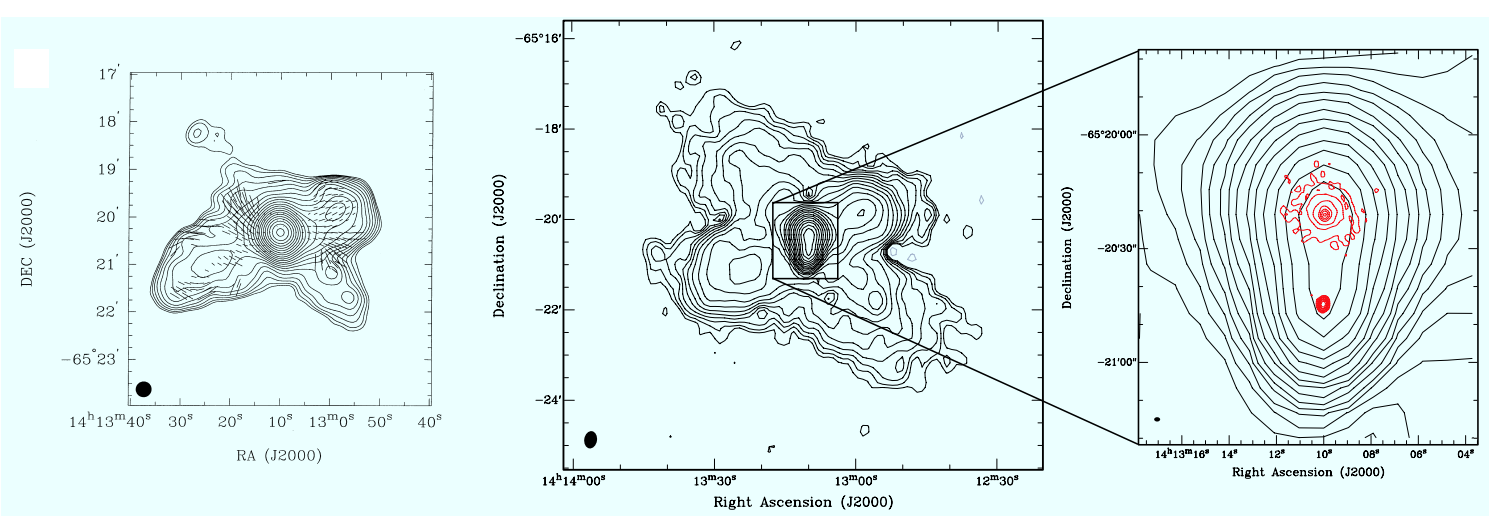

Figure 3: (Left) Image of Circinus made with five pre-CABB ATCA observations resulting in a sensitivity of $120 \mu \mathrm{Jy}_{\text {beam }}{ }^{-1}$ [1]. (Right) Image of Circinus made with a single CABB ATCA observation resulting in a sensitivity of $40 \mu \mathrm{Jy}_{\text {beam }}{ }^{-1}$ (Inset: SN 1996cr)

\subsection{Zoom modes}

Along with the new continuum modes, $\mathrm{CABB}$ has also introduced zoom modes for spectral line observing. The first zoom mode implemented, referred to as the "1 $\mathrm{MHz}$ zoom", provides the standard $2048 \times 1 \mathrm{MHz}$ continuum data plus $4 \times 1 \mathrm{MHz}$ zoom modes, each with $2048 \times 500 \mathrm{~Hz}$ channels each. Figure 4 shows a single baseline zoom spectrum, taken with $\mathrm{CABB}$, of a methanol maser. A "64 MHz zoom" is expected to be made available around October 2010, this mode will provide $32 \times 64 \mathrm{MHz}$ continuum channels and $2048 \times 32 \mathrm{KHz}$ channels. $4 \mathrm{MHz}$ and $16 \mathrm{MHz}$ zoom modes will follow in future.

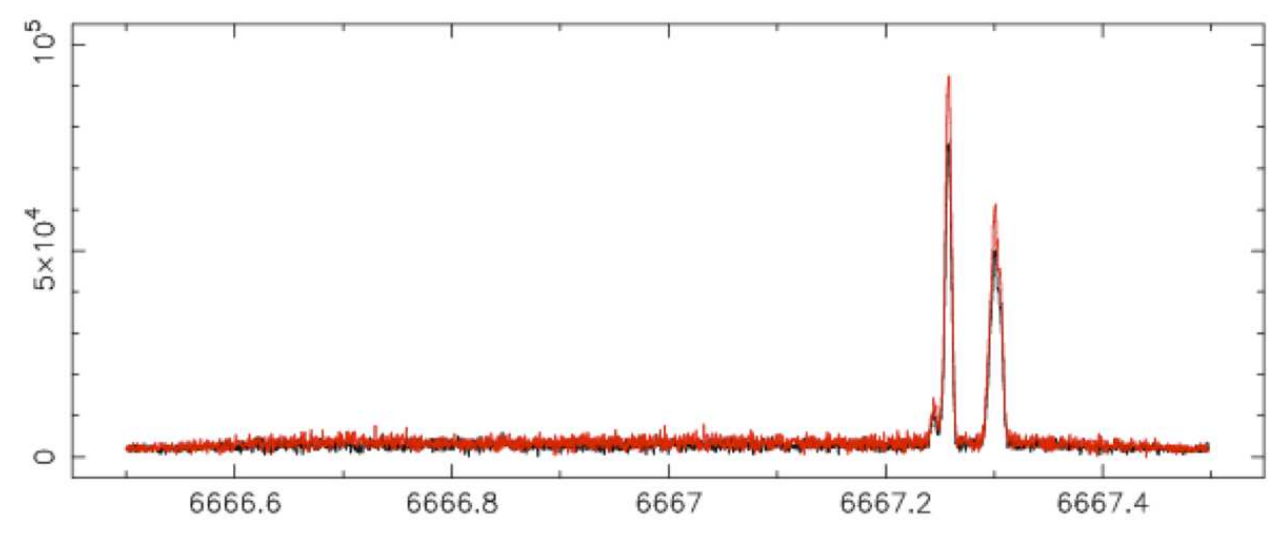

Figure 4: Single baseline zoom spectrum (2048 channels across $1 \mathrm{MHz}$ bandwidth) of a methanol maser. 


\subsection{Updated tools}

With the introduction of CABB a number of changes were required in the standard ATCA data reduction packaged, Miriad [2], to handle the larger number of channels. In addition to this, a number of improvements were made to the flagging tasks. Traditionally, the task BLFLAG was used to flag continuum data. With CABB data, this would be over-kill as each visibility flagged in BLFLAG would, by default, flag all 2048 channels. A new task, PGFLAG, was developed to improve spectral flagging, it replaces the older and more troublesome TVFLAG. PGFLAG allows the user to graphically view and flag bad data across both time and frequency space. An automatic flagger has also been developed, mirflag, building upon its predecessor PiFlag [3] by improving efficiency and performance. The new flagger has a number of flagging strategies and can now work, to some extent, on uncalibrated data by estimating and removing the effects of the bandpass and also amplitude variations over time. To aid in the visualisation of CABB data, a companion tool plotvis, was written to allow visibility data to be viewed interactively in $3 \mathrm{D}$, see Figure 2 . Both mirflag and plotvis are available for download at http://www.atnf.csiro.au/people/ Emil.Lenc/ATNF_Homepage/Tools.html.

\subsection{Check and double-check}

As can be seen in Figure 2, visibilities can vary dramatically with time and frequency for sources that exhibit structure. Sources previous deemed to be good calibrators, through narrowband snapshot surveys, may turn out to be less than ideal when observed with the wider bands of CABB. For this reason, it is important to visually inspect visibilities of your calibrator sources, or better yet, image them. Never assume that your calibrator source is unresolved! On a similar note, do not rely on pre-CABB scripts to process CABB data. It is important to understand and check all data reduction steps before attempting to automate the process.

\subsection{Going deep}

Classic CLEAN does not work well with extended sources and can detrimentally affect the final image fidelity. This is further exacerbated by the difficulty of performing selfcal with the ATCA. With only six antennas (15 baselines) in an east-west configuration, selfcal requires a particularly good input model before it will begin to converge on a good solution. This can be helped somewhat by modelling complex sources with Gaussian components in the $u-v$ plane. Another alternative is to use a multi-scale (CASA) or multi-resolution (AIPS) CLEAN. Furthermore, it is important to consider spectral effects in the CLEAN process - do not assume a flat spectral energy distribution (SED) for all of your source components. Currently, this can be achieved through MFCLEAN in Miriad (which fits a power-law SED to each pixel but does not handle sources with complex structure very well) or with multi-frequency, multi-scale CLEAN in CASA (which can fit a multi-term SED to each pixel and handles complex structures also). Figure 5 shows an example of where Gaussian model-fitting in the $u-v$ plane dramatically improved the dynamic range and fidelity of the final image.

When attempting to achieve high-dynamic range, it is important to check the wider field for confusing sources. These are not always immediately obvious when concentrating on your field of interest and are not always obvious when looking at source catalogues. For example, Figure 6 

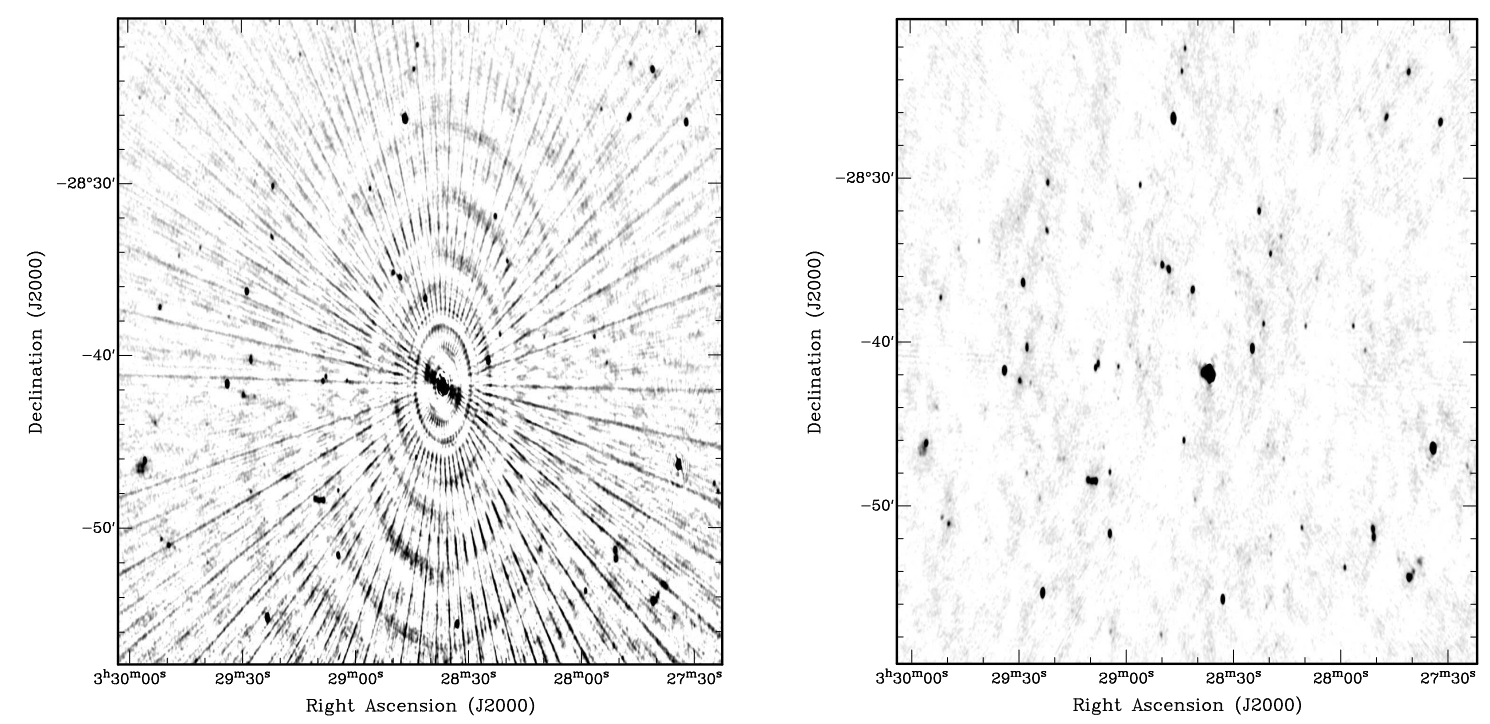

Figure 5: (a) Left, image of confusing source from first ATLAS data release. (b) Right, image with improved source modelling applied.

shows the field containing the calibrator source used for the ATLAS survey [4] using data combined from many epochs. The initial image had a higher than expected residual image noise but it was not immediately obvious where this additional noise originated from. By imaging successively larger fields-of-view it eventually became clear that one epoch was contaminated by a source approximately $20 \mathrm{deg}$ from the pointing centre - this source turned out to be the sun. When this one epoch was removed, the overall dynamic range improved by $40 \%$ and the image noise returned to the expected level.
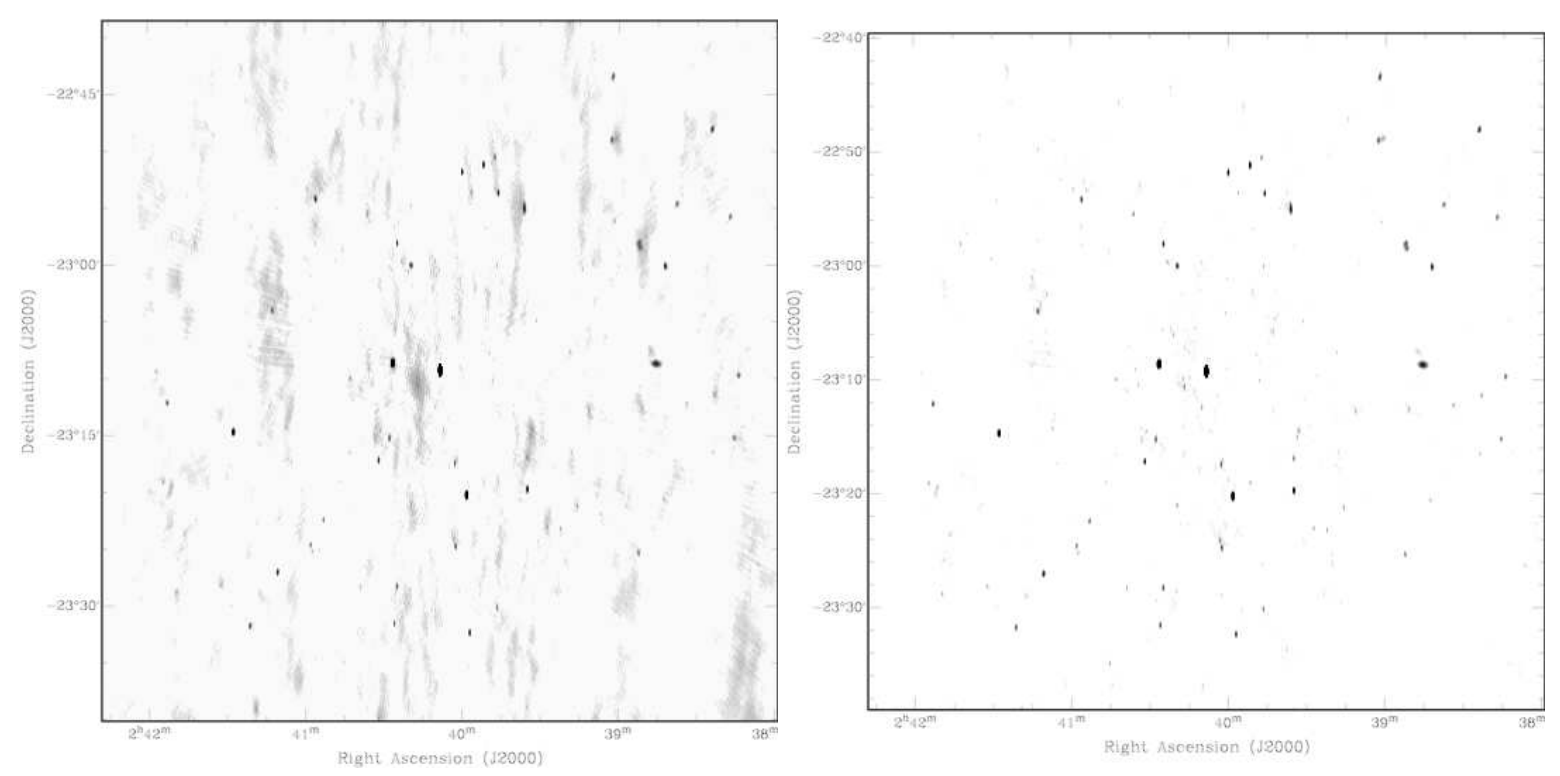

Figure 6: (a) Left, image of calibrator field used for ATLAS. (b) Right, field with solar interference removed. 


\subsection{Going wide}

An effect that needs to be considered for wide-field or mosaicked imaging is the frequency dependence of the primary beam. This is particularly significant at centimetre wavelengths where the fractional bandwidth is greatest. In general, if not corrected for, the effect causes an increased steepening of the spectral index for sources further from the pointing centre. Figure 7 shows how the SED for a hypothetical $1 \mathrm{Jy}$ flat-spectrum source (at $6 \mathrm{~cm}$ ) varies as it is progressively shifted from the centre of the field to a position 7 arcmin from the centre.

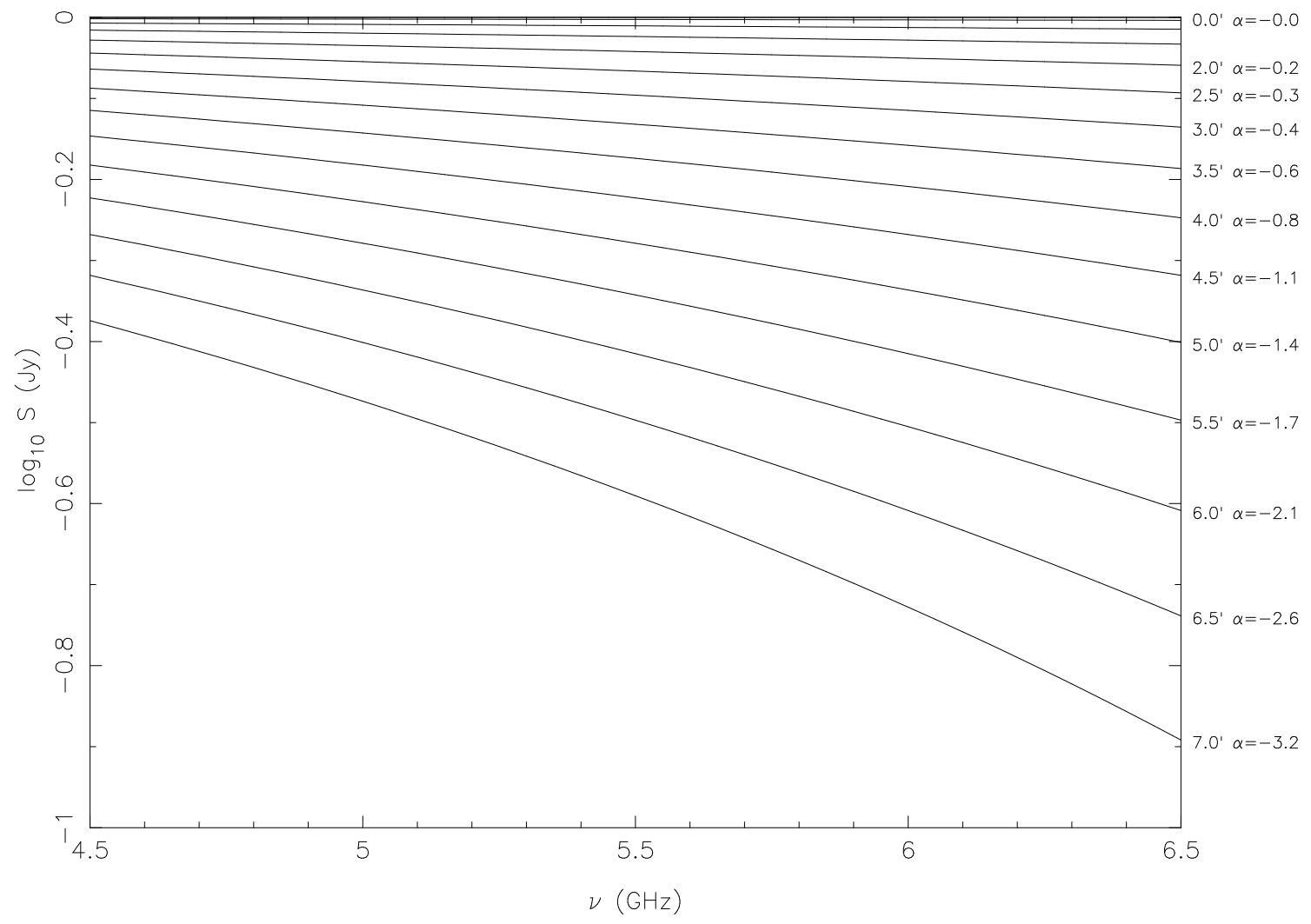

Figure 7: The SED of a hypothetical 1 Jy flat-spectrum source when it is placed at various distances (between 0 and $7 \mathrm{arcmin}$ ) from the centre of a $6 \mathrm{~cm}$ primary beam.

A first order solution to this problem is to split the observed band into smaller sub-bands. Each sub-band is inverted and deconvolved separately. By minimising the fractional bandwidth of each sub-band it is possible to apply a standard primary beam correction to each deconvolved image. The downside of this approach is that the sensitivity available in each sub-band is less than if entire band is used.

\subsection{Other challenges}

A number of other challenges still remain. One problem (not as yet fully verified) is that of varying bandpasses. Currently it is believed that this is not a problem at $6 \mathrm{~cm}$, although some scientific commissioning observations showed possible variation at $3 \mathrm{~cm}$ and subsequent observations at millimetre wavelengths also suggested bandpass variations. While more careful investigations are needed to confirm this, in the interim, the Miriad task MFCAL has been updated to allow 
time-varying bandpasses to be corrected for when regular bandpass calibrator observations are scheduled.

The wide bandwidths available with CABB have highlighted other subtle effects that were previously not obvious. Delays, seen as a phase gradient across the band, are observed to vary with time, see Figure 8. These delays are most prominent on the longest baselines and are associated with atmospheric "seeing". While the delays are not significant they will have a slight affect on overall sensitivity.

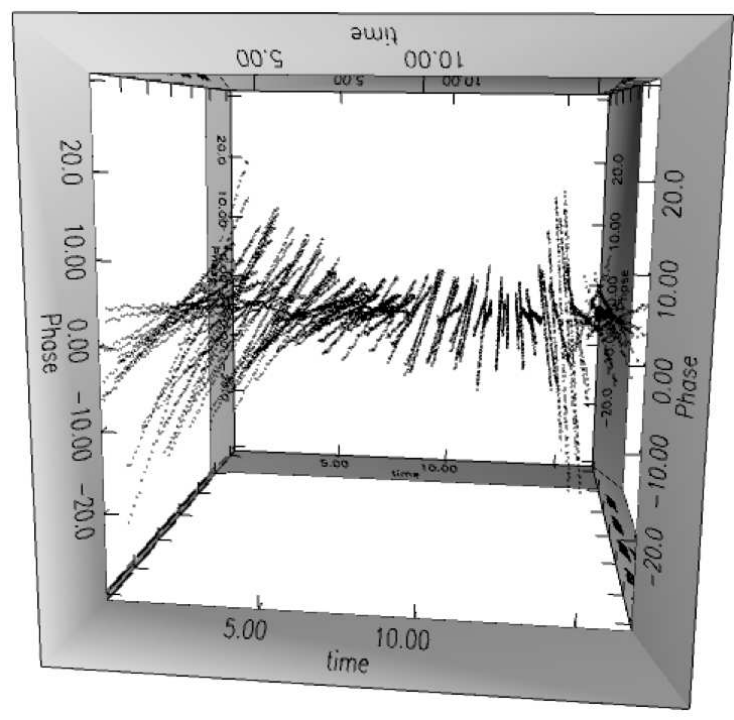

Figure 8: Phase gradients (delay) across the band vary with time and are associated with atmospheric "seeing".

Additional characteristics that vary across the band include the system temperature and the XY-phase. The system temperature, for example, can change by a factor of two from one end of the band to the other. Currently, Miriad only applies a single channel-independent correction for both the system temperature and the XY-phase. While both parameters are measured and available in $\mathrm{CABB}$ data files on a per-channel basis, the tools needed to apply the appropriate frequencydependent corrections still need to be updated in Miriad.

\section{The Long Baseline Array (LBA) and VLBI survey science}

The Long Baseline Array (LBA) consists of six antennas: the 70 m NASA Deep Space Network (DSN) antenna at Tidbinbilla; the $64 \mathrm{~m}$ antenna of the Australia Telescope National Facility (ATNF) near Parkes; the array of $6 \times 22 \mathrm{~m}$ antennas of the ATNF Australia Telescope Compact Array (ATCA) near Narrabri; the ATNF Mopra $22 \mathrm{~m}$ antenna near Coonabarabran; the University of Tasmania's $26 \mathrm{~m}$ antenna near Hobart; and the University of Tasmania's $30 \mathrm{~m}$ antenna near Ceduna. Recently, baselines to the first Australian Square Kilometre Array Pathfinder (ASKAP) antenna in Western Australia and an antenna near Warkworth in New Zealand have been demonstrated with the LBA, achieving baselines as long as $5500 \mathrm{~km}$. 


\subsection{VLBI Survey (Wide-Field) Capabilities}

Recent successes in wide-field VLBI imaging have demonstrated the possibility of VLBI survey science through the imaging, at full VLBI resolution and sensitivity, of multiple sources within the primary beam. Multiple-epoch VLBI observations of nearby starbursts, for example, have been used to monitor the formation and evolution of supernova remnants within these galaxies $[5,6]$, see Figure 9.
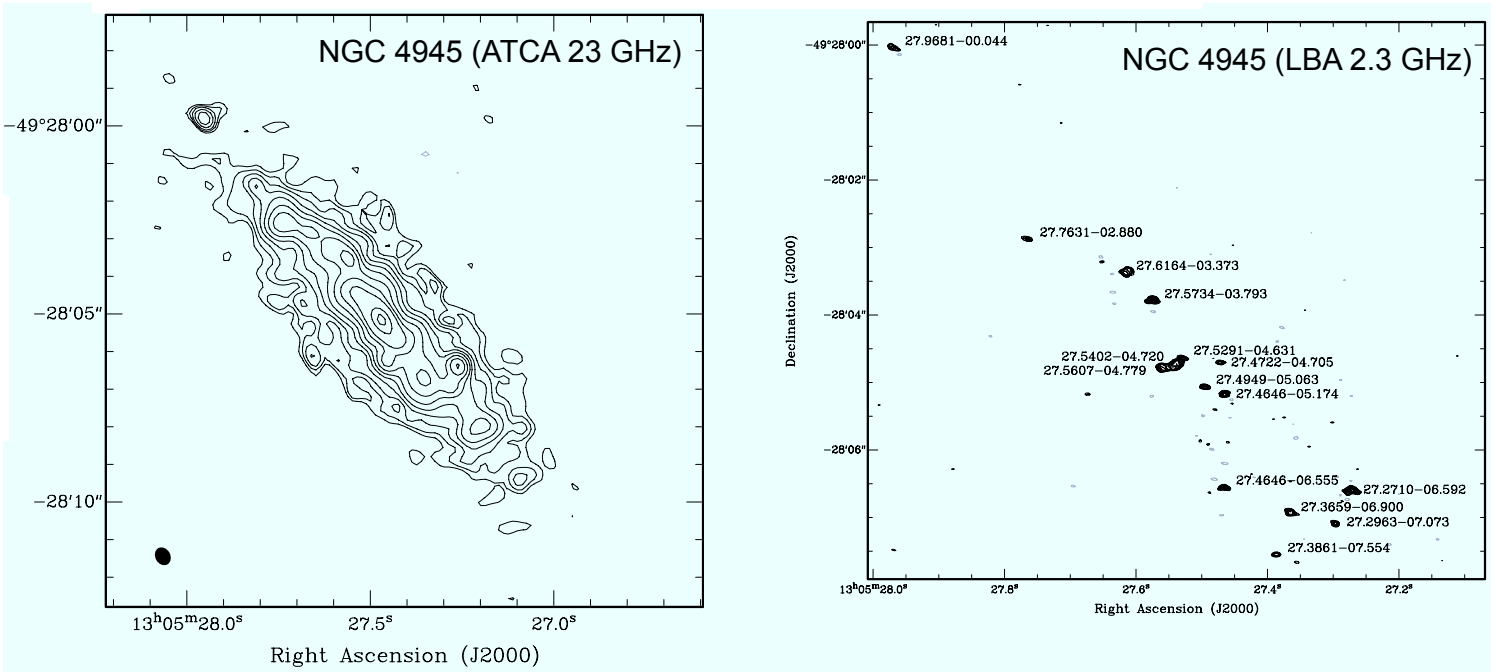

Figure 9: (Left) $23 \mathrm{GHz}$ ATCA Observation of the starburst galaxy NGC 4945. (Right) $2.3 \mathrm{GHz} \mathrm{LBA}$ Observation of NGC 4945 showing a population of supernova remnants.

Where possible, in-beam calibration can be used to maximise sensitivity and calibration of the target source. This technique has been used to observe AGN-jet hot spots in Centaurus A by using its nucleus as a calibrator [7], Figure 10. Where the calibrator source is found to have structure, a new task has been patched into difmap [8], cordump, has been developed to calibrate against such sources.

In the past, VLBI surveys required many passes of the correlator - a process that was time consuming. More recently, with clustered software correlators and high performance hardware correlators, another alternative is to correlate in a single pass using fine spectral and temporal resolution. Phase shifting, using the AIPS task UVFIX, can then be used to target specific sources or regions within the field without the need to recorrelate the data [9]. This method is important for untargeted observations, such as those of transients and supernovae, since their location cannot be determined apriori. The downside of this approach is that it results in large data files, processing is slow and the AIPS geometric delay models are simplistic and introduce astrometric errors. While the astrometric errors are difficult to overcome, recursive algorithms are currently being developed to greatly improve the imaging performance for large fields-of-view (Wucknitz et al., in prep).

For targeted surveys, a recent update to the DiFX Software Correlator [10] allows it to efficiently correlate multiple phase centres simultaneously. This method maintains excellent astrometry compared to that of AIPS/UVFIX since DiFX uses sophisticated geometric models from CALC. Additional benefits are that it results in smaller data files (since high spectral and temporal resolution is not required), full sensitivity and resolution is maintained over the entire field, and it has 


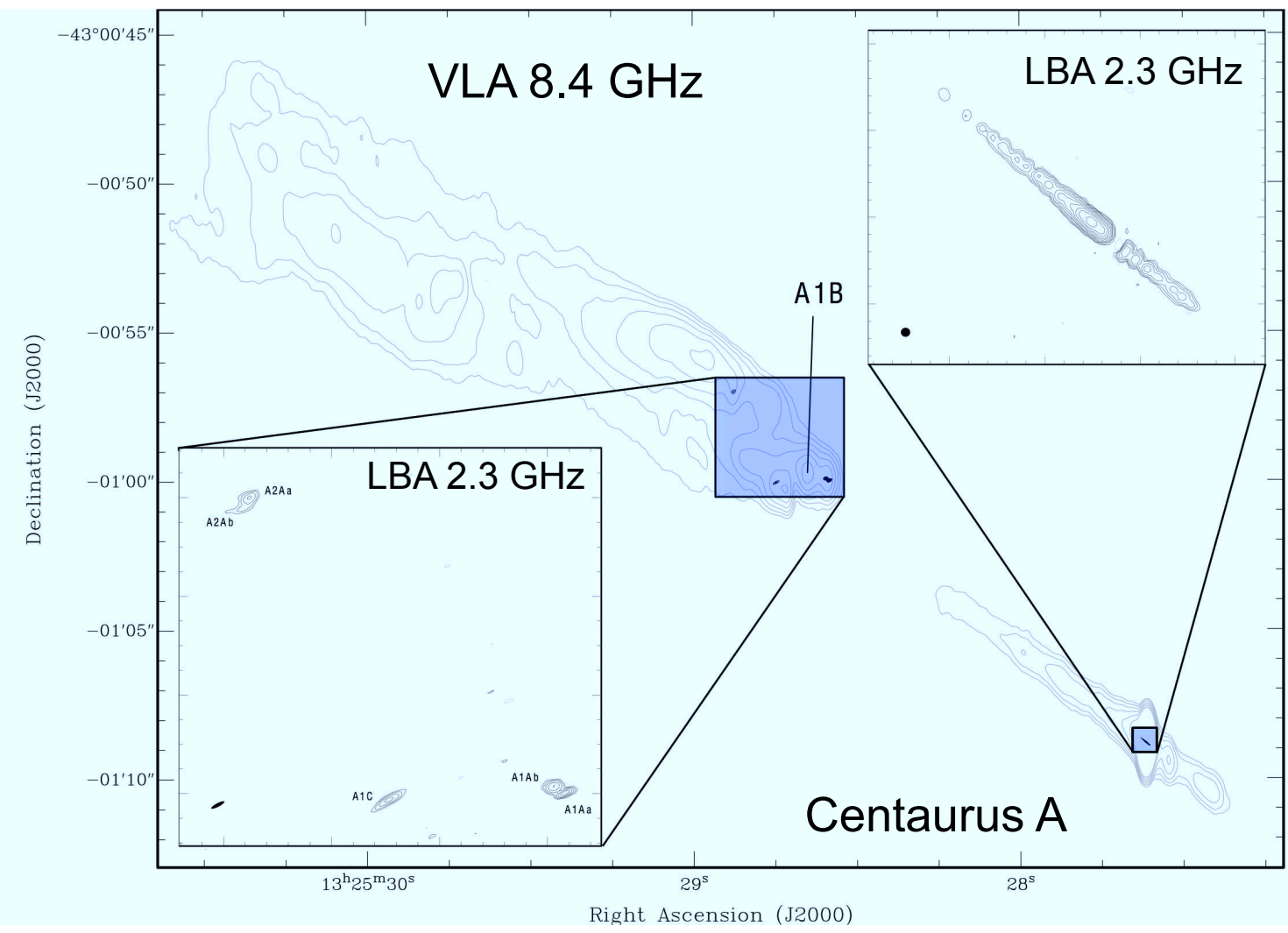

Figure 10: Centaurus A shown at low resolution with the VLA at $8.4 \mathrm{GHz}$ and at high resolution with the LBA at $2.3 \mathrm{GHz}$ using a single pointing to image both the AGN-jet (inset top-right) and jet-hotspots (inset bottom-left).

only a factor of a few processing overhead for $\sim 100$ pointings compared to performing a singlepointing correlation. This technique has been used in a VLBI survey of the Chandra Deep Field South field using the VLBA (Middelberg et al. 2010, in prep.) which targeted 96 sources, using the ATLAS CDFS source catalogue used as guide [4], and detected 21.

\section{Sharing experience}

Over recent years there have been a large number of complex instruments coming online. Often these instruments are in a state of flux as they go through a commissioning stage. While the instruments continue to record data, observers are exploring new domains with ill defined processes and documentation that is lagging behind - they are effectively "beta" testers. In such circumstances, there are typically "power" users at the facility but they are generally only known by locals and not necessarily by external users. Knowledge of the system is often distributed amongst a number of individuals and published papers documenting this knowledge are few and far between (even when available, the techniques section of the paper is often neglected or watered down). A solution was needed to help improve communication. 


\subsection{The ATCA Forum}

In general, the radio astronomy community is quite sociable and happy to help. What is usually missing is the avenue to provide this help. Our solution, for the CABB-Miriad upgrade, was to introduce an Internet forum - the "ATCA Forum". The forum is visible to all and has a persistent history so that others can share in the learning experience. It helps to release some load from the experts because they no longer need to be asked the same questions over and over again. The forum enables CABB-Miriad users to tap into a widely distributed knowledgebase on a wide range of topics e.g. Observing, calibration, imaging, deep observations, dynamic range, SEDs, variability, polarisation, spectral lines, etc. It provides a means of informing users of new features, changes to the system and/or new "challenges" that have been identified. As most CABB-Miriad "power" users keep track of new posts to the forum it allows new users, without a knowledge of who the power users are, to post undirected questions so that those that are best able to answer their questions can do so.

\subsection{Where now?}

The ATCA forum was initially set up as an experiment to gauge how well it would be received by a small number of test participants. We are currently in the process of incorporating user feedback so that we can make the forum more attractive to a larger community and integrate it into CSIRO Astronomy and Space Science. Our hope is that the forum can be expanded to include a wider range of facilities e.g. Parkes, Mopra, LBA and Tidbinbilla. By doing so it may enable a cross-pollination of ideas across different domains e.g. single-dish, spectral line, millimetre observing, VLBI, etc. Looking further into the future, there is no reason why the forum should be limited to our own facilities. Many of the problems we face are common to other facilities around the world. It would be ideal if the radio astronomy as a whole could get together in one place to discuss problems and ideas.

Before taking steps to further increase the scope of the forum it is important to ensure that users are comfortable in its use. In particular, it is important to identify any blockers that may discourage some users from contributing to the forum. e.g. techno-phobia or the fear of asking what they may believe to be "stupid" questions.

\section{References}

[1] M., Elmouttie, et al. 1998, mnras, 297, 1202

[2] R. J. Sault, P. J. Teuben, \& M. C. H. Wright 1995, ADASS IV, 77, 433

[3] E. Middelberg 2006, PASA, 23, 64

[4] R. P. Norris, et al. 2006, $A J, 132,2409$

[5] E. Lenc, \& S. J. Tingay 2006, AJ, 132, 1333

[6] E. Lenc, \& S. J. Tingay 2009, AJ, 137, 537

[7] S. J. Tingay \& E. Lenc 2009, $A J, 138,808$

[8] M. C. Shepherd, T. J. Pearson, \& G. B. Taylor 1994, BAAS, 26, 987 
[9] E. Lenc, et al. 2008, ApJ, 673, 78

[10] A. T. Deller, et al. 2007, PASP, 119, 318 